\title{
A self-learning TLBO based dynamic economic/environmental dispatch considering multiple plug-in electric vehicle loads
}

\author{
Zhile YANG, Kang LI ( $₫)$, Qun NIU, \\ Yusheng XUE, Aoife FOLEY
}

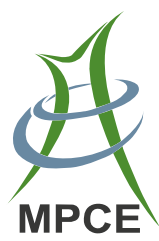

\begin{abstract}
Economic and environmental load dispatch aims to determine the amount of electricity generated from power plants to meet load demand while minimizing fossil fuel costs and air pollution emissions subject to operational and licensing requirements. These two scheduling problems are commonly formulated with non-smooth cost functions respectively considering various effects and constraints, such as the valve point effect, power balance and ramp rate limits. The expected increase in plug-in electric vehicles is likely to see a significant impact on the power system due to high charging power consumption and significant uncertainty in charging times. In this paper, multiple electric vehicle charging profiles are comparatively integrated into a 24-hour load demand in an economic and environment dispatch model. Self-learning teaching-learning based optimization (TLBO) is employed to solve the non-convex non-linear dispatch problems. Numerical results on well-known benchmark functions, as well as test systems with different scales of generation units show the significance of the new scheduling method.
\end{abstract}

Keywords Economic dispatch, Environmental dispatch, Plug-in electric vehicle, Self-learning, Teaching learning

CrossCheck date: 20 November 2014

Received: 8 October 2014/ Accepted: 25 November 2014/Published online: 16 December 2014

(C) The Author(s) 2014. This article is published with open access at Springerlink.com

Z. YANG, K. LI, School of Electrical, Electronics and Computer

Science, Queen's University Belfast, Belfast BT9 5AH, UK

(凹) e-mail: k.li@qub.ac.uk

Z. YANG

e-mail: zyang07@qub.ac.uk

Q. NIU, Shanghai Key Laboratory of Power Station Automation

Technology, School of Mechatronic Engineering and

Automation, Shanghai University, Shanghai 200072, China

Y. XUE, NARI Group Corporation (State Grid Electric Power

Research Institute), Nanjing 211106, China

A. FOLEY, School of Mechanical and Aerospace Engineering,

Queen's University, Belfast BT9 5AH, UK based optimization, Peak charging, Off-peak charging, Stochastic charging

\section{Introduction}

One of the key operational activities in the power system is to schedule power production according to the predicted load demands. Dynamic economic and environmental dispatches (DEED) are both crucial objectives in this scheduling task because a small percentage improvement may potentially bring significant cost savings and operational improvements $[1,2]$. The goal of economic dispatch is to minimize the cost by determining the power production of thermal power plant units, while managing system constraints, balancing power production and load demand, and meeting plant operational requirements, e.g. ramp rates. Similarly, the objective of environmental dispatch is to minimize emissions under the same system constraints. Both dispatch problems are difficult to solve due to the nonsmooth non-convex DEED formulations in cost functions and constraints, especially for large power systems [3].

Fast development of renewable power sources and changes in load demand bring more planning and operational uncertainties to the grid [4]. Plug-in electric vehicles (PEVs) with high penetrations are potentially important participants in the power system due to the additional large load requirements $[5,6]$. The stochastic charging of PEVs may also significantly affect the distribution grid as well as increase the generation costs and pollutants emissions if not managed efficiently. It is therefore of importance to measure the impact of different PEV charging scenarios on the power system and intelligently schedule and dispatch power generation using an optimized DEED system approach. 
In this paper, four different PEV charging scenarios are modeled using charging time probability distribution, based on PEV charging data from Electric Power Research Institute (EPRI), a full peak charging scenario and a full off-peak charging scenario and a stochastic charging scenario. These four charging time probability distributions are measured with a certain number of charging PEVs and integrated in the power demand of a 5-unit system and a 15-unit system respectively. Both the economic and environmental impacts are evaluated by solving the dynamic dispatch problems.

The objective functions and constraints of DEED problems are significantly non-smooth and non-convex due to factors like the valve point effects and exponential nature of emission output curve. Meta-heuristic approaches have consequently been employed to solve these problems. Numerous meta-heuristic algorithms have been used to solve the economic dispatch problem such as particle swarm optimization (PSO) [7], differential evolution (DE) [8], harmony search (HS) [9], biogeography-based optimization (BBO) [10], and imperialist competitive algorithm (ICA) [11], etc.

Based on our previous work [12], a new variant of teaching learning based optimization (TLBO), namely selflearning teaching-learning based optimization (SL-TLBO) is proposed and adopted to solve DEED problems. Some state-of-the-art variants of TLBO have been numerically compared to 10 well-known benchmarks and DEED problem for 5-unit and 15-unit power systems respectively. The dispatch results of four charging scenarios are also comparatively studied. The results show the significance of the new SL-TLBO scheduling method when applied to both benchmarks test and two scales of DEED test systems in terms of both the convergence speed and accuracy. For the four charging profiles, the comparative studies show that the off-peak charging scenario is the most economical and an environmental friendly choice.

\section{Problem formulations}

The DEED problem is a multi-objective problem combining the economic dispatch objective denoted as $F_{1}$ and environmental dispatch objective denoted as $F_{2}$. This twoobjective problem can also be transformed into a single objective problem as

$\min F=\omega_{e e} F_{1}+\left(1-\omega_{e e}\right) F_{2}$

where $\omega_{e e}$ is a weighting factor, being a constant between 0 and 1. The overall cost $F$ denotes the single objective to be minimized. In terms of the formulations of $F_{1}$ and $F_{2}$, both non-linear models for economic and environmental dispatch are considered.
2.1 Dynamic economic load dispatch model

The dynamic economic load dispatch problem is to minimize the total economic cost of the fossil fuel in a whole day time. The decision variable is the dispatched power $P_{i, t}$ in each time interval $t$. The problem is formulated as

$$
\begin{aligned}
F_{1}= & \sum_{t=1}^{T} \sum_{i=1}^{N_{u}} F_{i}\left(P_{i, t}\right)=\sum_{t=1}^{T} \sum_{i=1}^{N_{u}}\left[\left(a_{i}+b_{i} P_{i, t}+c_{i} P_{i, t}^{2}\right)\right] \\
& +\left|e_{i} \sin \left(f_{i}\left(P_{\text {imin }}-P_{i, t}\right)\right)\right|
\end{aligned}
$$

where $a_{i}, b_{i}$ and $c_{i}$ are the fuel cost coefficients of the $i^{\text {th }}$ generator; $e_{i}$ and $f_{i}$ the fuel cost coefficients for evaluating ripples in the cost curve caused by the valve-point effect; and $F_{1}$ accumulates the cost of $N_{u}$ generators in totally $T$ intervals. In addition to the cost functions, there are several system constraints associating with the objective functions as follows.

1) Power output limits:

$P_{i \min } \leq P_{i, t} \leq P_{i \max }\left(i=1,2, \ldots, N_{u}\right)$

where the power output should be within the capacity of each specific power generator $P_{\text {imax }}$ and $P_{\text {imin }}$.

2) Power balance limits:

$\sum_{i=1}^{N_{u}} P_{i, t}=P_{D, t}+P_{L, t+}+L_{e v, t} \quad t=1,2, \ldots, T$

The total power generated in each time interval should meet the power load demand in the corresponding time period. In this paper, the power demand constraint considers the original load demand $P_{D, t}$ in the time interval $t$, associated with the transmission losses $P_{L, t}$ and the PEV charging load $L_{e v, t}$. This PEV charging load is a new load type and will be further addressed in Section 3. The transmission losses are also considered and approximated with the widely used B-coefficients method [13] denoted as

$$
\begin{array}{r}
P_{L, t}=\sum_{i=1}^{N_{u}} \sum_{j=1}^{N_{u}} P_{i, t} B_{i, j} P_{j, t}+\sum_{i=1}^{N_{u}} B_{0, i} P_{i, t}+B_{00} \\
t=1,2, \ldots, T
\end{array}
$$

where $B_{i, j}, B_{0, i}$ and $B_{00}$ are loss coefficients. The handling approach is implemented by the method proposed in [14].

3) Ramp rate limits:

$\left\{\begin{array}{l}P_{i, t}-P_{i, t-1} \leq U R_{i} \\ P_{i, t-1}-P_{i, t} \geq D R_{i}\end{array}\right.$ 
Thermal generators are subject to the power ramp rate limitation that the power outputs cannot dramatically change between two adjacent intervals. The $D_{R i}$ and $U_{R i}$ are the ramp-up and ramp-down rate limits of the $i^{\text {th }}$ generator respectively. The dispatched power of a generator in the $t^{\text {th }}$ time interval $P_{i, t}$ should be limited by the previously dispatched power $P_{i, t-1}$ at time interval $t-1$ within rampup and ramp-down rate limits $D_{R i}$ and $U_{R i}$.

\subsection{Dynamic environmental load dispatch model}

The environmental load dispatch problem minimizes the emissions of environment pollutants including sulphur dioxide $\left(\mathrm{SO}_{2}\right)$ and nitrogen oxides $\left(\mathrm{NO}_{\mathrm{x}}\right)$ [15]. A quadratic polynomial formulation is associated with an exponential term to model the emissions as

$$
\begin{aligned}
F_{2}= & \sum_{t=1}^{T} \sum_{i=1}^{N_{u}} F_{i}\left(P_{i, t}\right)=\sum_{t=1}^{T} \sum_{i=1}^{N_{u}}\left[\left(\alpha_{i}+\beta_{i} P_{i, t}+\gamma_{i} P_{i, t}^{2}\right)\right] \\
& +\eta_{i} \exp \left(\delta_{i} P_{i, t}\right)
\end{aligned}
$$

where $\alpha_{i}, \beta_{i}, \gamma_{i}, \eta_{i}$, and $\delta_{i}$ are the emission coefficients of the $i^{\text {th }}$ generation unit. The power output limit, balance limit as well as the ramp rate limit is also taken into consideration in forming the constraints.

\section{Plug-in electric vehicle load profiles}

Plug-in electric vehicles are an unusual power demands and unlike traditional household and industry loads, the simultaneous charging of the $20 \mathrm{~kW}$ household chargers and $120 \mathrm{~kW}$ superchargers will possibly form huge ripples or even spikes on the daily power demand curve. Such effects could be avoided by the coordination and scheduling of charging. With the development of smart grid technology the introduction of smart PEV chargers to coordinate and control PEV charging looks highly alike. In this section, four different charging scenarios, including an EPRI predicted profile based on the assuming behaviors of drivers, an off-peak charging profile and a peak charging profile and a stochastic charging profile are modeled to compare and evaluate the impact on both economic and environmental aspects for the power system operation.

\subsection{EPRI profile}

The Electric Power Research Institute (EPRI) is a US funded non-profit organization founded in 1973. It is one of the leading organisations in the world, which produces publications and reports on the electric power industry. In an environmental assessment of PEVs [16], EPRI proposes an aggregate distribution of charging profiles to assess GHG emissions, where a probability distribution of charging profile is proposed as shown in Table 1.

In this charging scenario, over $60 \%$ power is delivered in 7 hours in the evening during 22:00 to 4:00. The other time slots see low charging rates and cover the rest of energy delivery.

\subsection{Off-peak profile}

In [17], two charging scenarios, peak and off-peak charging by assuming flat load demand for PEV charging during peak and off-peak time in the whole Ireland are proposed as a case study. The probability distribution in each hour for the off-peak case is illustrated in Table 2.

This profile assumes three charging levels with $18.5 \%$ of power is delivered every hour during 23:00 to $02: 00,9 \%$ of power is delivered in each time interval from 02:00 to 04:00 and the rest of charging is completed in 06:00. It is apparently an ideal case that besides the 8 hours charging, other time slots are forbidden for PEVs to get charged.

\subsection{Peak profile}

Similar to off-peak charging, peak charging assumes a flat load curve with three levels of charging power to describe a certain number of EVs getting charged during the peak load time on the wholesale electricity market. The probability distribution of each hour for the peak case is listed in Table 3.

This is another extreme case where all the PEV charging power requests are provided during peak time of electricity consumption during daytime from 13:00 to 20:00. The charging period and power in this paper are based on [16], where the peak time for charging is slightly shifted due to the profile of the load fleet.

Table 1 EPRI charging probability distribution

\begin{tabular}{lrrrrrr}
\hline Time & \multicolumn{7}{c}{ Probability/\% } & & & & \\
\hline 01:00-06:00 & 10 & 10 & 9.5 & 7 & 5 & 3 \\
07:00-12:00 & 1 & 0.3 & 0.3 & 1.3 & 2.1 & 2.1 \\
13:00-18:00 & 2.1 & 2.1 & 2.1 & 1 & 0.5 & 0.5 \\
19:00-24:00 & 1.6 & 3.6 & 5.4 & 9.5 & 10 & 10 \\
\hline
\end{tabular}

Table 2 Off-peak charging probability distribution

\begin{tabular}{lrrrrrr}
\hline Time & \multicolumn{1}{c}{ Probability/\% } & & & \\
\hline $01: 00-06: 00$ & 18.5 & 18.5 & 9 & 9 & 4 & 4 \\
$07: 00-12: 00$ & 0 & 0 & 0 & 0 & 0 & 0 \\
13:00-18:00 & 0 & 0 & 0 & 0 & 0 & 0 \\
19:00-24:00 & 0 & 0 & 0 & 0 & 18.5 & 18.5 \\
\hline
\end{tabular}


Table 3 Peak charging probability distribution

\begin{tabular}{lrrrrrr}
\hline Time & \multicolumn{7}{c}{ Probability/\% } \\
\hline 01:00-06:00 & 0 & 0 & 0 & 0 & 0 & 0 \\
07:00-12:00 & 0 & 0 & 0 & 0 & 0 & 0 \\
13:00-18:00 & 18.5 & 18.5 & 18.5 & 18.5 & 9 & 9 \\
19:00-24:00 & 4 & 4 & 0 & 0 & 0 & 0 \\
\hline
\end{tabular}

\subsection{Stochastic profile}

Considering the uncertainties of drivers' charging behavior, a stochastic charging profile is proposed in this paper. The stochastic load profile simulates some urgent group charging or distributed fast charging at random time throughout the whole day. The random probability follows the normal distribution with the mean value as $5 \%$. The probability distribution of each hour for the stochastic case is presented in Table 4.

The probability of stochastic charging profile in each hour ranges from $1.1 \%$ to $9.7 \%$. It changes randomly regardless of the peak or off-peak load time.

The four different PEV load charging distributions are illustrated in Fig. 1 respectively. These four profiles will impose extra load $L_{e v, t}$ in the power demand constraints (3). The new dispatch problems are tightly constrained and show strong non-convex, calling for more powerful computational tools to solve.

Table 4 Stochastic charging probability distribution

\begin{tabular}{lllllll}
\hline Time & \multicolumn{7}{c}{ Probability/\% } \\
\hline 01:00-06:00 & 5.7 & 4.9 & 4.8 & 2.4 & 2.6 & 9.7 \\
07:00-12:00 & 8.7 & 4.8 & 1.1 & 3.2 & 2.1 & 5.7 \\
13:00-18:00 & 3.8 & 2.2 & 2.1 & 6.1 & 3.2 & 2.2 \\
19:00-24:00 & 2.8 & 2.2 & 5.5 & 2.5 & 3.5 & 8.2 \\
\hline
\end{tabular}

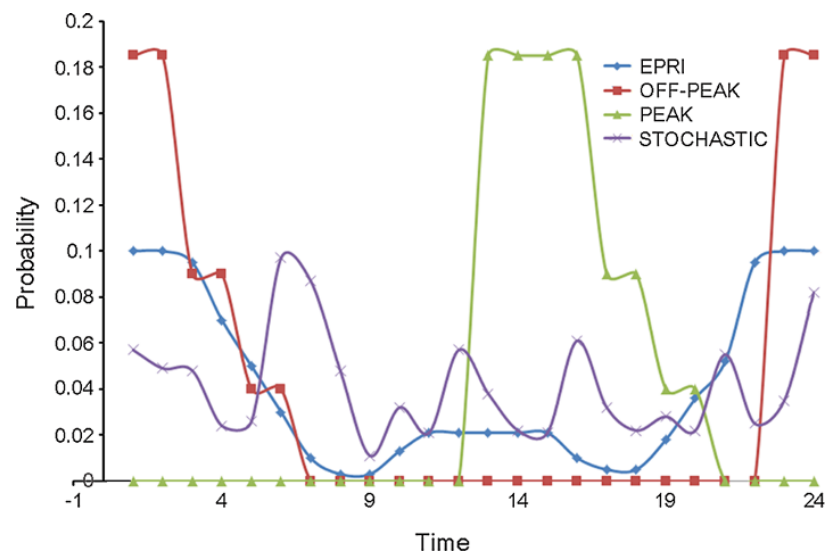

Fig. 1 Four different PEV load distributions

\section{Self-learning teaching-learning based optimization}

Teaching-learning based optimization (TLBO) is a new meta-heuristic algorithm proposed in 2011 and has been utilized in solving some engineering problems [18], [19], [20], [21]. Two phases are designed for each evolution iteration in the original TLBO, named teaching phase and learning phase respectively. Both the convergence speed and exploitation ability of TLBO have been tested on wellknown benchmarks and its effectiveness have been confirmed $[18,19]$. Though TLBO is powerful in solving many optimization problems, it can be further improved for specific problems. In this paper, a new self-learning phase is incorporated into original TLBO, aiming to continuously improve the exploitation ability.

\subsection{Teaching phase}

The teaching phase mimics a class teaching process that a teacher shares his/her knowledge to the students. A teacher will be first selected from the whole population by sorting the fitness function value. The deviation between the teacher and the mean of students will be calculated as: $D M_{i}=\operatorname{rand}_{1}\left(T_{i}-T_{F}\right.$ Mean $\left._{i}\right)$

where $D M_{i}$ is the value difference in the $i^{\text {th }}$ iteration; Mean $_{i}$ the mean value; $T_{i}$ the selected teacher; and $T_{F}$ a teaching factor. According to the original paper of TLBO, the $T_{F}$ can either be 1 or 2 denoted as

$T_{F}=\operatorname{round}\left(1+\operatorname{rand}_{2}(0,1)\right)$

Each learner in the class will gain knowledge from the value difference and update themselves as

$X_{i j}^{\text {new }}=X_{i j}^{\text {old }}+D M_{i}$

where $X_{i j}^{\text {new }}$ and $X_{i j}^{\text {old }}$ are the $j^{\text {th }}$ old and new learners of $i^{\text {th }}$ iteration. The new learners will compete with his/her predecessor and replace them if a better fitness value is achieved.

\subsection{Learning phase}

Followed the teaching phase, a learning phase provides a chance for each student to learn from a classmate. In this phase, each solution would randomly select another solution to compare the fitness, and update the knowledge storage according to the interaction. The phase is denoted as

$X_{i j}^{\text {new }}=\left\{\begin{array}{l}X_{i j}^{\text {old }}+\operatorname{rand}_{3}\left(X_{i k}-X_{i j}\right) f\left(X_{i k}\right)<f\left(X_{i j}\right) \\ X_{i j}^{\text {old }}+\operatorname{rand}_{3}\left(X_{i j}-X_{i k}\right) f\left(X_{i j}\right)<f\left(X_{i k}\right)\end{array}\right.$

where the $j^{\text {th }}$ learner $X_{i j}$ and $k^{\text {th }}$ learner $X_{i k}$ are randomly selected from the population in the $i^{\text {th }}$ iteration. Through a competition, the initial learner $X_{i j}$ will refresh his/her knowledge based on the deviation of the two learners. 
Similar with teaching phase, the refreshed student will have to compete with his/her predecessor and the better one will remain in the class.

\subsection{Self-learning phase}

The original TLBO performs well on weakly constrained or completely unconstrained problems. For strong constrained ill-conditioned optimization problems, the global optimal solutions are sensitive to slight changes. It is likely that the global optimum would be missed out due to the low exploitation ability. A self-learning phase is designed to further exploit the space near the particle position for the promising global optimum. Each student will get a chance to learn from his/her self-surrounding spaces. The self-learning phase is illustrated as

$X_{i j}^{\text {new }}=X_{i j}^{\text {old }}\left(1+\left(\right.\right.$ rand $\left.\left._{4}-0.5\right) \omega\right)$

where a self-learning weight $\omega$ is designed to determine the self-learning range of each particle. The parameter tuning of this factor will be discuss in Section 5 .

\section{Numerical experiments}

In order to evaluate the performance of the new SLTLBO algorithm, 10 well-known benchmark functions with 30 dimensions from [22] were tested. The SL-TLBO was compared with some TLBO variants including original TLBO, elite TLBO [23], and a modified TLBO [24]. In addition, some other commonly used algorithms which include weighted PSO [25], PSO-CF [26] and classical DE/ rand/1/bin [27] are selected for the comparative study.

\subsection{Benchmark functions}

The 10 benchmark functions are defined in [22] and listed with the dimensions and boundaries respectively as follows.

Sphere function $\left(f_{1}\right)$ : dimension $=30,[-100,100]$; Schwefel's problem $1.2\left(f_{2}\right)$ : dimension $=30$, $[-100$, 100];

Rosenbrock function $\left(f_{3}\right)$ : dimension $=30,[-30,30]$; Ackley's function $\left(f_{4}\right)$ : dimension $=30,[-32,32]$; Griewank function $\left(f_{5}\right)$ : dimension $=30,[-600,600]$; Rastrigin function $\left(f_{6}\right)$ : dimension $=30,[-5.12,5.12]$; Step function $\left(f_{7}\right)$ : dimension $=30,[-100,100]$; Schwefel's problem $2.21\left(f_{8}\right)$ : dimension $=30,[-100,100]$; Schwefel's problem $2.26\left(f_{9}\right)$ : dimension $=30,[-500$, 500];

Quartic function $\left(f_{10}\right)$ : dimension $=30,[-1.28,1.28]$.

\subsection{Determination of control parameters in SL-TLBO}

Though there are no algorithm specific parameters to be tuned in original TLBO, the new SL-TLBO method has introduced a self-learning weighting factor $\omega$ to adjust the learning range. It is therefore important to find proper settings of $\omega$. Three benchmarks have been tested with the $\omega$ ranging from 0.1 to 10 . The maximum generation is set as 100 and the particle number is 30 . To eliminate the experimental incidents, 30 different run were employed. The initialization values were randomly generated within the boundary and were taken as the same input for all the algorithms. The searching results are showed in Table 5 with mean values and standard deviations for each parameter setting respectively.

Table 5 Benchmark tests result with different weighting factor

\begin{tabular}{lllr}
\hline & $f_{1}$ & $f_{4}$ & $f_{9}$ \\
\hline$\omega=0.1$ & $8.4878 \mathrm{e}-27 \pm 7.9217 \mathrm{e}-26$ & $2.3488 \mathrm{e}-11 \pm 3.7617 \mathrm{e}-12$ & $-5.0731 \mathrm{e} 03 \pm 2.6459 \mathrm{e} 03$ \\
$\omega=0.3$ & $2.5354 \mathrm{e}-29 \pm 3.0583 \mathrm{e}-28$ & $6.5648 \mathrm{e}-13 \pm 2.7191 \mathrm{e}-12$ & $-5.0010 \mathrm{e} 03 \pm 2.7628 \mathrm{e} 03$ \\
$\omega=0.2$ & $1.1154 \mathrm{e}-27 \pm 1.0138 \mathrm{e}-26$ & $1.9191 \mathrm{e}-13 \pm 6.9575 \mathrm{e}-13$ & $-4.9442 \mathrm{e} 03 \pm 2.5526 \mathrm{e} 03$ \\
$\omega=0.4$ & $2.2029 \mathrm{e}-30 \pm 4.5483 \mathrm{e}-29$ & $3.5113 \mathrm{e}-14 \pm 1.5700 \mathrm{e}-13$ & $-5.0013 \mathrm{e} 03 \pm 2.2619 \mathrm{e} 03$ \\
$\omega=0.5$ & $5.0894 \mathrm{e}-32 \pm 6.1376 \mathrm{e}-31$ & $9.6515 \mathrm{e}-15 \pm 3.3226 \mathrm{e}-14$ & $-4.9387 \mathrm{e} 03 \pm 2.3824 \mathrm{e} 03$ \\
$\omega=0.6$ & $1.1328 \mathrm{e}-33 \pm 8.3986 \mathrm{e}-33$ & $4.6777 \mathrm{e}-15 \pm 4.8539 \mathrm{e}-15$ & $-5.1075 \mathrm{e} 03 \pm 2.6581 \mathrm{e} 03$ \\
$\omega=0.7$ & $9.4206 \mathrm{e}-35 \pm 1.5798 \mathrm{e}-33$ & $4.4409 \mathrm{e}-15 \pm 0.0000$ & $-5.3365 \mathrm{e} 03 \pm 2.4129 \mathrm{e} 03$ \\
$\omega=0.8$ & $4.3055 \mathrm{e}-37 \pm 3.3442 \mathrm{e}-36$ & $4.3225 \mathrm{e}-15 \pm 3.4930 \mathrm{e}-15$ & $-5.0814 \mathrm{e} 03 \pm 2.7728 \mathrm{e} 03$ \\
$\omega=0.9$ & $1.6973 \mathrm{e}-38 \pm 1.7813 \mathrm{e}-37$ & $4.4409 \mathrm{e}-15 \pm 0.0000$ & $-5.0571 \mathrm{e} 03 \pm 2.7343 \mathrm{e} 03$ \\
$\omega=1$ & $1.8046 \mathrm{e}-40 \pm 3.4272 \mathrm{e}-39$ & $4.4409 \mathrm{e}-15 \pm 0.0000$ & $-5.3451 \mathrm{e} 03 \pm 3.8784 \mathrm{e} 03$ \\
$\omega=2$ & $4.5126 \mathrm{e}-78 \pm 3.4475 \mathrm{e}-79$ & $8.8816 \mathrm{e}-16 \pm 0.0000$ & $-5.4113 \mathrm{e} 03 \pm 2.3002 \mathrm{e} 03$ \\
$\omega=3$ & $8.2091 \mathrm{e}-89 \pm 1.6473 \mathrm{e}-87$ & $8.8816 \mathrm{e}-16 \pm 0.0000$ & $-5.0689 \mathrm{e} 03 \pm 3.1955 \mathrm{e} 03$ \\
$\omega=5$ & $1.0296 \mathrm{e}-68 \pm 2.9208 \mathrm{e}-67$ & $8.8816 \mathrm{e}-16 \pm 0.0000$ & $-4.9982 \mathrm{e} 03 \pm 2.5672 \mathrm{e} 03$ \\
$\omega=10$ & $2.1032 \mathrm{e}-48 \pm 6.0272 \mathrm{e}-47$ & $8.8816 \mathrm{e}-16 \pm 0.0000$ & $-5.2526 \mathrm{e} 03 \pm 2.5658 \mathrm{e} 03$ \\
\hline
\end{tabular}


It could be observed that the results get better with the increase of $\omega$ in both on $f_{1}$ and $f_{4}$. The best results are achieved when $\omega$ is 3 in the $f_{1}$ test and from 1 to 10 in the $f_{4}$ test. On the other hand, the final results change stochastically with the increase of $\omega$ in the $f_{9}$ test where the best result was achieved when $\omega$ is 2 . These results show that the performance of the parameter settings for the new SLTLBO algorithm is problem specific. We choose $\omega=3$ for the further comparative study on algorithms performance in sub-section 5.3 .

\subsection{Simulation results and discussions}

In this sub-section, the new SL-TLBO algorithm were tested and comparatively studied with some counterparts on the ten benchmark functions tests mentioned in 5.1. To fairly compare the algorithm performance, the number of the function evaluation (FES) is introduced as the iteration criteria. Each generation of PSO and DE method accounts for one FES. The original TLBO has two phases and accounts for double times of function evaluation, whereas the SL-TLBO has one more self-learning phase in each generation and triple times are used to calculate the FES.

In terms of the parameters settings for the algorithms, the population number is 30 while the FES is set as 12,000 . The weighted PSO uses $c_{1}=1, c_{2}=3, w_{\max }=0.9, w_{\min }=0.4$; for PSO-CF, $c_{1}=c_{2}=2.05, K=0.729$; for classical DE, $F=0.7, C R=0.5$; for elite TLBO, the elite number is set as 5 ; the self-learning weighting factor $\omega$ is 3 ; no specific parameters are required to be set for original TLBO and mTLBO. Similarly, 30 different runs were carried out for each benchmark function by each algorithm. The mean values and standard deviations for each test are illustrated in Table 6.

From the results, it is shown that the new SL-TLBO performs best in the limited FES among 7 of totally 10 tests. In the tests on $f_{1}, f_{4}, f_{7}$, and $f_{8}$, the SL-TLBO significantly outperforms other counterparts. Comparable performance for the new algorithm is displayed on $f_{2}, f_{5}, f_{6}$. In the cases of $f_{3}, f_{9}$, and $f_{10}$, SL-TLBO is outperformed by the original TLBO, mTLBO, and eTLBO, respectively.
However, in these three cases, the performances of the four TLBO variants are relatively the same, which indicates that the general problem solving capability of SL-TLBO appears to be satisfactory. Therefore, this new method is utilized in solving the DEED problem with PEV loads.

\section{Simulation results on DEED problem and discussions}

\subsection{Case 1: 5-unit economic dispatch without PEV}

In order to show the significance of the new SL-TLBO algorithm, the original 5-unit considering valve-point effect and transmission loss is tested. The FES is set as 60000, and the population number for the SL-TLBO method is 50 . The 5-unit economic dispatch benchmark data is taken from [28]. According to the experimental tests, $\omega$ is set as 0.05 , which is much smaller than the previous setting in benchmark functions tests. This is because the DEED problem is highly constrained due to which a big learning rate will easily cause the violation of constraints. On the other hand, a small learning factor would increase the searching ability in nearby solution space, which is more adaptable for DEED problem to search better solutions in a limited available space.

It can be seen from the Table 7 that the new SL-TLBO can obtain the best results comparing with some previous methods. This method is then utilized in PEV integration analysis.

\subsection{Case 2: 5-unit economic dispatch with PEV}

In this case, a 5-unit system combined with the four PEV load profiles considering valve-point effect and transmission losses is investigated to dynamically dispatch the generation production from the economic perspective.

Considering the local population and load situation of the benchmark system, an extra load of 30000 different types of PEVs is integrated, where $45 \%$ of these PEVs are low hybrid vehicles equipped with $15 \mathrm{kWh}$ batteries.

Table 6 Benchmark tests results for different algorithms

\begin{tabular}{|c|c|c|c|c|c|c|c|}
\hline & wPSO & PSO-CF & $\mathrm{DE}$ & TLBO & eTLBO & mTLBO & SL-TLBO \\
\hline$f_{1}$ & $4.869 \mathrm{e} 02 \pm 1.069 \mathrm{e} 03$ & $1.904 \mathrm{e} 03 \pm 3.895 \mathrm{e} 03$ & $3.562 \mathrm{e} 03 \pm 1.130 \mathrm{e} 04$ & $2.090 \mathrm{e}-45 \pm 2.617 \mathrm{e}-44$ & $2.774 \mathrm{e}-76 \pm 2.905 \mathrm{e}-75$ & $8.200 \mathrm{e}-91 \pm 1.521 \mathrm{e}-89$ & $2.747 \mathrm{e}-115 \pm 4.087 \mathrm{e}-114$ \\
\hline$f_{2}$ & $5.155 \mathrm{e} 00 \pm 5.236 \mathrm{e} 01$ & $4.390 \mathrm{e}-02 \pm 5.259 \mathrm{e}-01$ & $1.601 \mathrm{e}-01 \pm 1.690 \mathrm{e} 00$ & $0.000 \mathrm{e} 00 \pm 0.000 \mathrm{e} 00$ & $5.342 \mathrm{e}-31 \pm 1.268 \mathrm{e}-29$ & $0.000 \mathrm{e} 00 \pm 0.000 \mathrm{e} 00$ & $0.000 \mathrm{e} 00 \pm 0.000 \mathrm{e} 00$ \\
\hline$f_{3}$ & $1.369 \mathrm{e} 03 \pm 1.128 \mathrm{e} 04$ & $1.714 \mathrm{e} 00 \pm 1.074 \mathrm{e} 01$ & $3.829 \mathrm{e} 00 \pm 9.934 \mathrm{e} 00$ & $1.547 \mathrm{e} 00 \pm 7.442 \mathrm{e} 00$ & $3.077 \mathrm{e} 00 \pm 5.274 \mathrm{e} 00$ & $3.513 \mathrm{e} 00 \pm 3.118 \mathrm{e} 00$ & $1.746 \mathrm{e} 00 \pm 4.955 \mathrm{e} 00$ \\
\hline$f_{4}$ & $2.114 \mathrm{e} 01 \pm 2.957 \mathrm{e}-01$ & $1.946 \mathrm{e} 01 \pm 1.398 \mathrm{e} 01$ & $1.446 \mathrm{e} 01 \pm 4.263 \mathrm{e} 01$ & $2.828 \mathrm{e}-08 \pm 8.340 \mathrm{e}-07$ & $2.072 \mathrm{e}-15 \pm 9.173 \mathrm{e}-15$ & $9.063 \mathrm{e} 00 \pm 5.678 \mathrm{e} 01$ & $8.882 \mathrm{e}-16 \pm 0.000 \mathrm{e} 00$ \\
\hline$f_{5}$ & $2.493 \mathrm{e} 01 \pm 3.151 \mathrm{e} 01$ & $1.390 \mathrm{e} 01 \pm 9.142 \mathrm{e}-01$ & $1.491 \mathrm{e} 01 \pm 4.792 \mathrm{e} 00$ & $0.000 \mathrm{e} 00 \pm 0.000 \mathrm{e} 00$ & $0.000 \mathrm{e} 00 \pm 0.000 \mathrm{e} 00$ & $0.000 \mathrm{e} 00 \pm 0.000 \mathrm{e} 00$ & $0.000 \mathrm{e} 00 \pm 0.000 \mathrm{e} 00$ \\
\hline$f_{6}$ & $2.297 \mathrm{e} 02 \pm 2.516 \mathrm{e} 02$ & $1.092 \mathrm{e} 02 \pm 8.724 \mathrm{e} 01$ & $4.953 \mathrm{e} 01 \pm 2.736 \mathrm{e} 02$ & $4.970 \mathrm{e}-08 \pm 1.466 \mathrm{e}-06$ & $0.000 \mathrm{e} 00 \pm 0.000 \mathrm{e} 00$ & $0.000 \mathrm{e} 00 \pm 0.000 \mathrm{e} 00$ & $0.000 \mathrm{e} 00 \pm 0.000 \mathrm{e} 00$ \\
\hline$f_{7}$ & $2.076 \mathrm{e} 01 \pm 4.834 \mathrm{e} 01$ & $7.076 \mathrm{e} 00 \pm 1.700 \mathrm{e} 01$ & $1.229 \mathrm{e} 01 \pm 2.125 \mathrm{e} 01$ & $5.084 \mathrm{e} 00 \pm 3.918 \mathrm{e} 00$ & $5.546 \mathrm{e} 00 \pm 4.097 \mathrm{e} 00$ & $5.878 \mathrm{e} 00 \pm 3.798 \mathrm{e} 00$ & $4.744 \mathrm{e} 00 \pm 3.225 \mathrm{e} 00$ \\
\hline$f_{8}$ & $2.751 \mathrm{e} 00 \pm 2.513 \mathrm{e} 00$ & $1.089 \mathrm{e} 00 \pm 1.370 \mathrm{e} 00$ & $2.170 \mathrm{e} 00 \pm 3.321 \mathrm{e} 00$ & $5.162 \mathrm{e}-24 \pm 2.490 \mathrm{e}-23$ & $2.568 \mathrm{e}-39 \pm 3.930 \mathrm{e}-38$ & $1.022 \mathrm{e}-46 \pm 9.416 \mathrm{e}-46$ & $1.881 \mathrm{e}-59 \pm 3.248 \mathrm{e}-58$ \\
\hline$f_{9}$ & $-4.321 \mathrm{e} 03 \pm 2.680 \mathrm{e} 02$ & $-5.223 \mathrm{e} 03 \pm 2.449 \mathrm{e} 03$ & $-5.898 \mathrm{e} 03 \pm 1.752 \mathrm{e} 03$ & $-6.083 \mathrm{e} 03 \pm 2.319 \mathrm{e} 03$ & $-5.667 \mathrm{e} 03 \pm 2.339 \mathrm{e} 03$ & $-6.382 \mathrm{e} 03 \pm 1.733 \mathrm{e} 03$ & $-6.302 \mathrm{e} 03 \pm 2.588 \mathrm{e} 03$ \\
\hline$f_{10}$ & $2.489 \mathrm{e} 01 \pm 3.211 \mathrm{e} 01$ & $1.571 \mathrm{e} 01 \pm 7.902 \mathrm{e} 00$ & $1.821 \mathrm{e} 01 \pm 6.346 \mathrm{e} 00$ & $9.465 \mathrm{e} 00 \pm 2.256 \mathrm{e} 00$ & $9.323 \mathrm{e} 00 \pm 2.601 \mathrm{e} 00$ & $9.429 \mathrm{e} 00 \pm 1.488 \mathrm{e} 00$ & $9.549 \mathrm{e} 00 \pm 2.747 \mathrm{e} 00$ \\
\hline
\end{tabular}


Besides, $25 \%$ of PEVs are medium hybrid vehicles using $25 \mathrm{kWh}$ batteries, and $30 \%$ PEVs are pure battery vehicles of which the total power are provided by $40 \mathrm{kWh}$ batteries as in Table 8. It is also assumed that $50 \%$ SOC are the energy necessity for PEVs in a 24-hour period [32]. The total PEV load for one day is calculated as $L_{e v}=$ $30000 \times(15 \times 45 \%+25 \times 25 \%+40 \times 30 \%) \times 0.5=$ 375 MW.

Fig. 2 illustrates the four different load demand profiles with corresponding situations of PEV charging probability in a 24-hour time period. Differences lie on the noon time between 12:00-17:00 and midnight between 22:00-06:00. The peak load has increased significantly in the peak charging situation shown as the purple line in Fig. 2 lasts till the next load valley. On the contrary, the valley load is

Table 7 Comparison of total fuel cost over 30 runs (Case 1: 5-unit without PEV)

\begin{tabular}{llll}
\hline Method & \multicolumn{2}{l}{ Fuel cost (\$/day) } & \\
\cline { 2 - 4 } & Min & Ave & Max \\
\hline SA [28] & 47356.00 & NA & NA \\
PS [29] & 46530.00 & NA & NA \\
EP [30] & 46777.00 & NA & NA \\
PSO [31] & 44253.24 & 45657.06 & 46402.52 \\
SL-TLBO & 44199.98 & 45655.74 & 46113.64 \\
\hline
\end{tabular}

Table 8 Multiple types of PEV

\begin{tabular}{lrr}
\hline EV type & Battery capacity/kWh & Proportion/\% \\
\hline Low hybrid & 15 & 45 \\
High hybrid & 25 & 25 \\
Pure battery & 40 & 30 \\
\hline
\end{tabular}

increased by both off-peak charging and EPRI charging (see green and red line in Fig. 2). The stochastic charging profile generates a small new sub-peak during 6:00 to 9:00 in the morning showed in the blue line again in Fig. 2.

The four circumstances are tested by seven algorithms respectively. The particle number is set as 50 and the FES is 20000. The algorithms parameter settings are almost the same with the tests in 5.3, while the only difference lies on the setting of the self-learning weighting factor $\omega$ in SLTLBO as mentioned in case 1 .

Table 9 shows the dispatched results of four PEV charging profiles solved by seven algorithms. The SLTLBO outperforms all its counterparts and achieves the best results on all the four charging profiles. Moreover, comparing the four charging patterns, the off-peak charging costs $46508.86 \$$ /day which is the lowest among all the situations. In contrast, the peak charging costs reach to 47367.17 \$/day and becomes the highest cost. The EPRI charging profile ranks the second lowest in terms of the cost and outperforms the stochastic charging behavior which ranks the third place with the costs of 46770.71 \$/ day and $47158.86 \$$ /day respectively. The off-peak charging profile costs 858.31 \$/day lower than the peak charging profile, which implies that under the same charging

Table 9 Economic dispatching result for case 1 (\$/day)

\begin{tabular}{lllll}
\hline & $L_{\text {EPRI }}$ & $L_{\text {Offp }}$ & $L_{\text {Peak }}$ & $L_{\text {Sto }}$ \\
\hline wPSO & 49004.13 & 48587.97 & 50875.78 & 49333.11 \\
PSO-CF & 51482.18 & 51231.77 & 51682.02 & 51292.57 \\
DE & 51457.32 & 51238.97 & 51310.22 & 51283.18 \\
TLBO & 49649.47 & 48884.45 & 48775.31 & 49292.38 \\
eTLBO & 49049.49 & 49306.12 & 49270.68 & 49549.59 \\
mTLBO & 48974.99 & 47656.89 & 48459.7 & 48970.59 \\
SL-TLBO & 46770.71 & 46508.86 & 47367.17 & 47158.86 \\
\hline
\end{tabular}

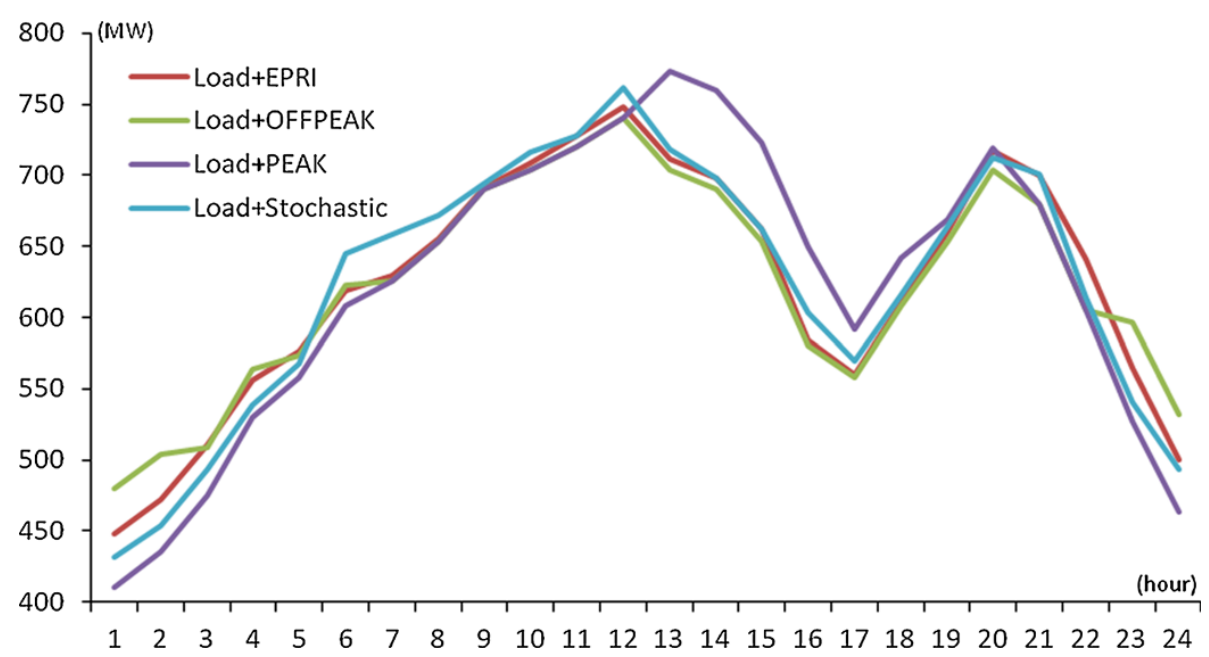

Fig. 2 Power load demands with four PEV profiles 
demand, charging preferentially at off-peak time could save $1.85 \%$ in terms of the economic cost.

\subsection{Case 3: 5-unit environmental dispatch with PEV}

In this case, the environmental emission is considered rather than the economic cost. Similarly, the 5-unit system is employed and the data is taken from [33]. The objective function is presented as (7) and the constraints are the same with economic dispatch in the previous subsection. The PEV number and the power necessity are the same with the aforementioned economic case. All the four charging profiles are also comparatively tested by seven algorithms including wPSO, PSO-CF, classical DE, original TLBO, elite TLBO, modified TLBO, and SL-TLBO. The parameter initializations for these algorithms are the same with case 2.

Table 10 shows the environmental dispatch results for the four different charging profiles solved by seven algorithms. The lowest environmental emission results are still produced by the proposed SL-TLBO method on all the four PEV charging scenarios. Among these charging profiles, the off-peak charging profile again emits the least air pollutants with the amount of $18659.24 \mathrm{lb} /$ day achieved by SL-TLBO, while the peak charging profile produces most emissions with $19227.18 \mathrm{lb} /$ day. Therefore, a reduction of $3.0 \%$ emission production with $567.94 \mathrm{lb}$ /day from the generation side would be achieved by shifting the PEV charging time. It is the same situation as in case 2, the EPRI charging profile and the stochastic charging profile rank the $2^{\text {nd }}$ and the $3^{\text {rd }}$ with the emissions of $18820.78 \mathrm{lb} /$ day and $18963.69 \mathrm{lb} /$ day respectively.

\subsection{Case 4: 5-unit economic and environmental dispatch with PEV}

Since the economic aspect and the environmental aspect are both vital for power system dispatch, a combination of these two objectives is also investigated. The $\omega_{\mathrm{ee}}$ in the objective function (1) is defined as 0.5 in this case to comprehensively consider the economic and environmental profit and trade-off the both situations. The 5-unit test system is again utilized of which the load demand and four PEV charging situations remain the same with previous cases. Dealing with the objective function with significant high non-linear characteristics with both sinusoidal and exponential terms, the case would be more complex for algorithms to solve. In order to evaluate the algorithms performances and compare the four charging situations, the seven algorithms with same parameter initializations are again implemented.

Table 11 shows all the dispatching results considering both economic and environmental aspects. Not
Table 10 Environmental dispatching result for case 2 (lb/day)

\begin{tabular}{lllll}
\hline & $L_{\text {EPRI }}$ & $L_{\text {Offp }}$ & $L_{\text {Peak }}$ & $L_{\text {Sto }}$ \\
\hline wPSO & 19189.83 & 18998.30 & 19443.99 & 19112.25 \\
PSO-CF & 20232.49 & 19794.18 & 20440.12 & 20183.56 \\
DE & 20030.88 & 19658.45 & 20493.63 & 20109.08 \\
TLBO & 19002.82 & 18887.37 & 19483.80 & 19195.84 \\
eTLBO & 19170.59 & 18930.36 & 19390.75 & 19154.89 \\
mTLBO & 19112.78 & 18879.95 & 19379.09 & 19369.38 \\
SL-TLBO & 18820.78 & 18659.24 & 19227.18 & 18963.69 \\
\hline
\end{tabular}

Table 11 Economic/environmental dispatching result for case 3 $(0.5 \cdot \$ /$ day $+0.5 \cdot \mathrm{lb} /$ day $)$

\begin{tabular}{lllll}
\hline & $L_{\text {EPRI }}$ & $L_{\text {Offp }}$ & $L_{\text {Peak }}$ & $L_{\text {Sto }}$ \\
\hline wPSO & 35785.15 & 34705.22 & 35514.07 & 36022.33 \\
PSO-CF & 36611.46 & 36601.88 & 36814.66 & 36632.76 \\
DE & 36534.69 & 36421.38 & 36657.76 & 36581.62 \\
TLBO & 35037.04 & 34959.16 & 35112.58 & 35269.76 \\
eTLBO & 35064.55 & 35167.21 & 35048.14 & 35654.87 \\
mTLBO & 35300.92 & 35355.49 & 35162.68 & 34857.65 \\
SL-TLBO & 33998.31 & 33924.62 & 34731.92 & 34245.83 \\
\hline
\end{tabular}

incidentally, SL-TLBO again achieved the best fitness values. The fitness value of off-peak charging profile is 33924.62 while it is 34731.92 for the peak charging scenario. There is a $2.4 \%$ deviation between these two values. If the performances of all the algorithms are compared in all the cases, SL-TLBO always gives the best performance.

\subsection{Case 5: 15-unit economic dispatch with PEV}

The 5-unit system is only a small scale system with 120 variables for a dynamic dispatch in 24-hour period of time. However, dispatching tasks for larger system widely exist. In this case, a 15-unit system is implemented of which the total load demand is $60981 \mathrm{MW}$ [34]. The number of variables is tripled and reaches to 360 in one-day time. To simplify the situation, the valve point-effect is neglected in the objective function as

$F=\sum_{t=1}^{T} \sum_{i=1}^{N_{u}} F_{i}\left(P_{i, t}\right)=\sum_{t=1}^{T} \sum_{i=1}^{N_{u}}\left[\left(a_{i}+b_{i} P_{i, t}+c_{i} P_{i, t}^{2}\right)\right]$

where the transmission loss and ramp rate constraints are also considered. The system data is taken from [31]. To evaluate the economic impact of the four PEV charging profiles in this test system, the total PEV number is proportionally increased to 90000. While the PEV types remain the same as in Table 8 , resulting in that the total $\mathrm{PEV}$ charging power necessity is tripled to $1,125 \mathrm{MW}$ in a single day. This extra PEV charging load is distributed 
Table 12 Economic dispatching result for Case 4 (\$/day)

\begin{tabular}{lllll}
\hline & $L_{\text {EPRI }}$ & $L_{\text {Offp }}$ & $L_{\text {Peak }}$ & $L_{\text {Sto }}$ \\
\hline wPSO & 783004.14 & 783650.51 & 783863.93 & 784610.33 \\
PSO-CF & 784391.24 & 784532.96 & 785851.62 & 785491.74 \\
DE & 784354.55 & 784313.52 & 785512.30 & 785273.31 \\
TLBO & 781644.49 & 783002.47 & 784004.33 & 783962.29 \\
eTLBO & 782323.93 & 782320.70 & 783383.72 & 783280.51 \\
mTLBO & 781562.91 & 781179.19 & 782922.74 & 782138.87 \\
SL-TLBO & 781001.23 & 780862.82 & 781961.91 & 781459.24 \\
\hline
\end{tabular}

within the four charging scenarios and integrated with the original load demand. The seven algorithms are again employed to solve the large scale problem for comparative study where the specific parameter settings for these algorithms are the same with those in case 2 .

The results provided in Table 12 illustrate that the SLTLBO method again achieved the optimal solutions, outperforming with all the other six algorithms in all the four load scenarios. The lowest cost appears in the off-peak charging scenario with the cost of $780862.82 \$ /$ day, while the peak charging scenario costs 1099.09 \$/day more than the off-peak one, reaching to $781961.91 \$ /$ day.

\section{Conclusion}

Dynamic economic dispatch has long been an intractable problem for power system operators and the complexity is ever increasing with new participants such as PEV entering in the equation. In this paper, the non-convex dynamic economic and environmental dispatch has been comparatively investigated with the integrations of various PEV charging scenarios. A new self-learning teaching learning based optimization method is proposed to solve the economic and environmental dispatch problems. A small scale 5-unit system and a large scale 15-unit system are tested in 24-hour time period. Four different PEV charging scenarios including EPRI predicted charging, off-peak charging, peak charging and stochastic charging profiles with different number of PEV have been integrated in the load demands of both systems. The numerical results show that the new SL-TLBO algorithm is a viable alternative approach for solving both small and large scale dynamic dispatch problems and outperforms other popular heuristic methods and state-of-the-art TLBO variants in the tests on well-known benchmarks and DEED problem with proper parameter tuning. In terms of the four PEV charging scenarios, the off-peak charging scenario, as expected, has the advantage in reducing the economic cost and environmental pollutant emissions.

In future studies, renewable energy sources such as photovoltaic panels and wind power as well as the vehicle to grid $(\mathrm{V} 2 \mathrm{G})$ will be introduced in the system dispatch to comprehensively analyze the interaction between the PEV and power systems.

Acknowledgments Zhile YANG would like to thank UK Engineering and Physical Sciences Research Council (EPSRC) for sponsoring his research. The authors would also like to thank UK EPSRC under grant EP/L001063/1 and China NSFC under grants 51361130153 and 61273040.

Open Access This article is distributed under the terms of the Creative Commons Attribution License which permits any use, distribution, and reproduction in any medium, provided the original author(s) and the source are credited.

\section{Reference}

[1] Wong KP, Fung CC (1993) Simulated annealing based economic dispatch algorithm. IEE Proc Gener Transm Distrib 140(6):509-515

[2] Victoire TAA, Jeyakumar AE (2005) Reserve constrained dynamic dispatch of units with valve-point effects. IEEE Trans Power Syst 20(3):1273-1282

[3] Abido MA (2003) Environmental/economic power dispatch using multiobjective evolutionary algorithms. IEEE Trans Power Syst 18(4):1529-1537

[4] Zhang Y, Yao F, Lu HHC et al (2013) Sequential quadratic programming particle swarm optimization for wind power system operations considering emissions. J Mod Power Syst Clean Energy 1(3):231-240

[5] Saber AY, Venayagamoorthy GK (2012) Resource scheduling under uncertainty in a smart grid with renewables and plug-in vehicles. IEEE Syst J 6(1):103-109

[6] Yang Z, Li K, Foley A et al (2014) Optimal scheduling methods to integrate plug-in electric vehicles with the power system: a review. In: Proceedings of the 19th world congress of the International Federation of Automatic Control (IFAC' 14), Cape Town, South Africa, 24-29 August 2014, pp 8594-8603

[7] Mohammadi-Ivatloo B, Rabiee A, Soroudi A et al (2012) Iteration PSO with time varying acceleration coefficients for solving non-convex economic dispatch problems. Int $\mathrm{J}$ Electr Power Energy Syst 42(1):508-516

[8] Yuan XH, Wang L, Zhang YC et al (2009) A hybrid differential evolution method for dynamic economic dispatch with valvepoint effects. Expert Syst Appl 36(2-2):4042-4048

[9] Niu Q, Zhang HY, Wang XH et al (2014) A hybrid harmony search with arithmetic crossover operation for economic dispatch. Int J Electr Power Energy Syst 62:237-257

[10] Bhattacharya A, Chattopadhyay PK (2010) Biogeography-based optimization for different economic load dispatch problems. IEEE Trans Power Syst 25(2):1064-1077

[11] Mohammadi-Ivatloo B, Rabiee A, Soroudi A et al (2012) Imperialist competitive algorithm for solving non-convex dynamic economic power dispatch. Energy 44(1):228-240

[12] Yang Z, Li K, Niu Q et al (2014) Non-convex dynamic economic/environmental dispatch with plug-in electric vehicle loads. In: Proceedings of the IEEE symposium series on computational intelligence (SSCI' 14, accepted)

[13] Wood AJ, Wollenberg BF (2012) Power generation, operation, and control. Wiley, New York 
[14] Niu Q, Zhang HY, Li K et al (2014) An efficient harmony search with new pitch adjustment for dynamic economic dispatch. Energy 65:25-43

[15] Gong DW, Zhang Y, Qi CL (2010) Environmental/economic power dispatch using a hybrid multi-objective optimization algorithm. Int J Electr Power Energy Syst 32(6):607-614

[16] EPRI executive summary: environmental assessment of plug-in hybrid electric vehicles, Volume 2: United States air quality analysis based on AEO-2006 assumptions for 2030 (2007). Electric Power Research Institute, Palo Alto

[17] Foley A, Tyther B, Calnan P et al (2013) Impacts of electric vehicle charging under electricity market operations. Appl Energy 101:93-102

[18] Rao RV, Savsani VJ, Vakharia DP (2011) Teaching-learningbased optimization: a novel method for constrained mechanical design optimization problems. Comput Aided Des 43(3):303-315

[19] Rao RV, Savsani VJ, Vakharia DP (2012) Teaching-learningbased optimization: an optimization method for continuous nonlinear large scale problems. Inf Sci 183(1):1-15

[20] Niu Q, Zhang HY, Li K (2014) An improved TLBO with elite strategy for parameters identification of PEM fuel cell and solar cell models. Int J Hydrog Energy 39(8):3837-3854

[21] Sultana S, Roy PK (2014) Optimal capacitor placement in radial distribution systems using teaching learning based optimization. Int J Electr Power Energy Syst 54:387-398

[22] Yao X, Liu Y, Lin GM (2013) Evolutionary programming made faster. IEEE Trans Evol Comput 3(2):82-102

[23] Rao RV, Patel V (2013) Comparative performance of an elitist teaching-learning-based optimization algorithm for solving unconstrained optimization problems. Int $\mathbf{J}$ Ind Eng Comput $4(1): 29-50$

[24] Satapathy SC, Naik A (2014) Modified teaching-learning-based optimization algorithm for global numerical optimization-a comparative study. Swarm Evol Comput 16:28-37

[25] Shi Y, Eberhart RC (1998) Parameter selection in particle swarm optimization, evolutionary programming VII. Springer, Berlin, pp 591-600

[26] Clerc M, Kennedy J (2002) The particle swarm-explosion, stability, and convergence in a multidimensional complex space. IEEE Trans Evol Comput 6(1):58-73

[27] Das S, Suganthan PN (2011) Differential evolution: a survey of the state-of-the-art. IEEE Trans Evol Comput 15(1):4-31

[28] Panigrahi CK, Chattopadhyay PK, Chakrabarti RN et al (2006) Simulated annealing technique for dynamic economic dispatch. Electr Power Compon Syst 34(5):577-586

[29] Alsumait JS, Qasem M, Sykulski JK et al (2010) An improved pattern search based algorithm to solve the dynamic economic dispatch problem with valve-point effect. Energy Convers Manag 51(10):2062-2067

[30] Basu M (2007) Dynamic economic emission dispatch using evolutionary programming and fuzzy satisfying method. Int $\mathrm{J}$ Emerg Electr Power Syst 8(4):1-15

[31] Hemamalini S, Simon SP (2011) Dynamic economic dispatch using artificial bee colony algorithm for units with valve-point effect. Eur Trans Electr Power 21(1):70-81

[32] Saber AY, Venayagamoorthy GK (2011) Plug-in vehicles and renewable energy sources for cost and emission reductions. IEEE Trans Ind Electron 58(4):1229-1238

[33] Wang LF, Singh C (2007) Environmental/economic power dispatch using a fuzzified multi-objective particle swarm optimization algorithm. Electr Power Syst Res 77(12):1654-1664
[34] Gaing ZL, Ou TC (2009) Dynamic economic dispatch solution using fast evolutionary programming with swarm direction. In: Proceedings of the 4th IEEE conference on industrial electronics and applications (ICIEA'09), Xi'an, China, 25-27 May 2009, pp 1538-1544

Zhile YANG is pursuing his Ph.D. degree at the School of Electrical, Electronics and Computer Science, Queen's University Belfast. He received the BEng in Electrical Engineering and the MSc degree in Control Theory and Control Engineering from Shanghai University (SHU) in 2010 and 2013 respectively. His research interests include non-linear modeling and meta-heuristic optimization on power system and electric vehicle.

Kang LI received the B.Sc. degree from Xiangtan University, Hunan, China, in 1989, the M.Sc. degree from the Harbin Institute of Technology, Harbin, China, in 1992, and the Ph.D. degree from Shanghai Jiaotong University, Shanghai, China, in 1995. He is currently a Professor of intelligent systems and control with the School of Electronics, Electrical Engineering, and Computer Science, Queen's University Belfast, Belfast, U.K. His research interests include nonlinear system modeling, identification, and control, bioinspired computational intelligence, fault diagnosis and detection, with recent applications on power systems and renewable energy, polymer extrusion processes, bioinformatics with applications on food safety, healthcare, and biomedical engineering.

Qun NIU is received the B.Sc. degree in automation and the Ph.D. degree in control theory and control engineering from the East China University of Science and Technology, Shanghai, China, in 2002 and 2007 respectively.

From 2009 to 2011, she was a Postdoctoral Research Fellow with Queen's University Belfast, Belfast, U.K. She is currently an Associate Professor with the Shanghai Key Laboratory of Power Station Automation Technology, School of Mechatronics and Automation, Shanghai University, Shanghai. Her main research interests include meta-heuristic optimization and intelligent computing with applications to power system scheduling and economic dispatch, and manufacturing scheduling.

Yusheng XUE received his Ph.D. degree in Electrical Engineering from the University of Liege (Belgium) in 1987. He became a Member of Chinese Academy of Engineering in 1995. He is now the Honorary President of State Grid Electric Power Research Institute (SGEPRI), State Grid Corporation of China. His research interests include nonlinear stability, control and power system automation.

Aoife FOLEY is a Lecturer in the School of Mechanical and Aerospace Engineering, Queen's University Belfast. She has a BE (Hons) (1996), a PhD (2011) from University College Cork and an MSc (Hons) (1999) from Trinity College Dublin. She was awarded an Environmental Protection Agency STRIVE Fellowship (2009) to model the impact of Electric Vehicles on GHG emissions. She has more than 12 year's industrial experience with ESB International, Siemens and SWS. She is a Fellow of Engineers Ireland (2011), a Member of the IEEE PES (2009) and Secretary of the IEEE VTS UK and Republic of Ireland Chapter (2011). She has authored and coauthored almost 50 peer reviewed technical publications. Her research interests include wind power integration using controllable loads and energy storage, electricity markets and power systems operations. 